\title{
Serum ferritin levels are associated with carotid atherosclerosis in Chinese postmenopausal women: the Shanghai Changfeng Study
}

\author{
Hui $\mathrm{Ma}^{1}$, Huandong $\mathrm{Lin}^{2}$, Yu Hu${ }^{1}$, Xiaoming $\mathrm{Li}^{2}$, Wanyuan $\mathrm{He}^{3}$, Xuejuan $\mathrm{Jin}^{4}$, Jian $\mathrm{GaO}^{5}$, Naiqing Zhao ${ }^{6}$, \\ Binbin Song ${ }^{7}$, Boshen $\mathrm{Pan}^{7}$ and Xin $\mathrm{GaO}^{2 *}$ \\ ${ }^{1}$ Department of Geriatrics, Zhongshan Hospital, Fudan University, Shanghai 200032, People's Republic of China \\ ${ }^{2}$ Department of Endocrinology and Metabolism, Zhongshan Hospital, Fudan University, Shanghai 200032, \\ People's Republic of China \\ ${ }^{3}$ Department of Ultrasonography, Zhongshan Hospital, Fudan University, Shanghai 200032, People's Republic of China \\ ${ }^{4}$ Clinical Epidemiology Center, Zhongshan Hospital, Fudan University, Shanghai 200032, People's Republic of China \\ ${ }^{5}$ Department of Clinical Nutrition, Zhongshan Hospital, Fudan University, Shanghai 200032, People's Republic of China \\ ${ }^{6}$ Department of Biostatistics, College of Public Health, Fudan University, Shanghai 200032, People's Republic of China \\ ${ }^{7}$ Department of Laboratory Medicine, Zhongshan Hospital, Fudan University, Shanghai 20032, People's Republic of China
}

(Submitted 16 January 2015 - Final revision received 6 May 2015 - Accepted 7 May 2015 - First published online 12 August 2015)

\begin{abstract}
Postmenopausal women are at increased risk of CVD: the increased serum ferritin level may be involved in the pathogenesis. The aim of the present study is to investigate the relationship of ferritin and carotid atherosclerosis in postmenopausal women. A total of 1178 postmenopausal women (mean age, 60.8 years) were enrolled from the Changfeng Study. A standard interview, anthropometric measurements and laboratory analyses were performed for each participant. Bilateral CIMT (carotid intima-media thickness) were measured using ultrasonography, and the presence of carotid plaques was assessed. Serum ferritin was measured using electrochemiluminescence immunoassay. The results showed that serum ferritin was 181.9 (SD 65.8$) \mathrm{ng} / \mathrm{ml}$ in the postmenopausal women. Multivariate, linear, stepwise regression analysis demonstrated that age (standardised $\beta=0 \cdot 233, P<0 \cdot 001$ ), alanine transaminase (standardised $\beta=0 \cdot 194$, $P<0.001$ ), $\log$ homeostasis model assessment index for insulin resistance (standardised $\beta=0 \cdot 181, P<0 \cdot 001$ ), TAG (standardised $\beta=0.083, P=0.003), \mathrm{Hb}$ (standardised $\beta=0.080, P=0.004)$ and PPG (2-h glucose levels following a 75 -g oral glucose challenge) (standardised $\beta=0.079, P=0.004$ ) were independently associated with serum ferritin. Compared with the ferritin level of subjects in the first quartile, that in the fourth quartile had greater CIMT, and higher prevalence of carotid plaque. After adjusting for conventional CVD risk factors, $\mathrm{Hb}$, leucocytes, log urine albumin:creatinine ratio and liver function, the ferritin level of postmenopausal women in the fourth quartile had a 1.587 -fold increased risk of carotid plaques relative to those in the lowest quartile. In conclusion, these results suggest that serum ferritin is independently and positively associated with carotid atherosclerosis in postmenopausal women and that ferritin may be implicated in atherosclerosis.
\end{abstract}

\section{Key words: Ferritin: Carotid intima-media thickness: Carotid plaques: Carotid atherosclerosis}

Postmenopausal women are at increased risk of CVD, which may be attributed to dyslipidemia, oxidative stress, loss of the protective effect of oestrogen, centralised adiposity, insulin resistance, and other factors ${ }^{(1)}$. The cessation of menses contributes to a general increase in body Fe, translating into elevated serum ferritin, as evidenced by a 3-fold greater ferritin in postmenopausal compared with premenopausal women ${ }^{(2)}$. Serum ferritin concentration is the best non-invasively measurable indicator of body Fe stores ${ }^{(3)}$. In 1981, Sullivan ${ }^{(3)}$ proposed for the first time that body $\mathrm{Fe}$ stores are positively related to CHD risk. In 1992, a Finnish study confirmed this hypothesis, showing a positive relationship between serum ferritin and risk of acute myocardial infarction in men ${ }^{(4)}$, after which interest in this theory started growing. The first prospective study in women was conducted in 11471 Dutch post-menopausal female subjects aged 49-70 years $^{(5)}$. In the study, the multivariate hazard ratio of ischemic strokes in the highest tertile of serum ferritin concentration was $2 \cdot 23$ (95\% CI $1.05,4 \cdot 73)$ compared to the lowest ${ }^{(5)}$. In a cross sectional study that included German men and

Abbreviations: CIMT, carotid intima-media thickness; HOMA-B\%, homeostasis model assessment index for $\beta$-cell function; HOMA-IR, homeostasis model assessment index for insulin resistance; PPG, 2-h blood glucose following a 75-g oral glucose challenge; sTfR, soluble transferrin receptor; UACR, urine albumin:creatinine ratio; WHR, waist:hip ratio.

*Corresponding author: X. Gao, fax +86 21 64037269, email happy20061208@126.com; gao.xin@zs-hospital.sh.cn 
women, there was an association of serum ferritin with carotid plaque prevalence in both men (OR 1.33; $95 \%$ CI 1.08, 1.44) and women (OR 1.29; $95 \%$ CI $0.98,1.75)^{(6)}$. However, there are also a number of other well-conducted studies that have found no association of markers of body Fe and risk of CVD. In a prospective study performed in a French population of 9917 subjects, the risk of IHD was not related to serum ferritin ${ }^{(7)}$. In a large, population-based prospective study, higher levels of ferritin were not associated with an increased risk of mortality among the Third National Health and Nutrition Examination Survey participants without a baseline history of CVD or cancer $^{(8)}$. Epidemiological studies have provided: contradictory results regarding Fe stores and subsequent atherosclerosis and coronary artery disease; little data on this relationship in Chinese population, the dietary habits and Fe intakes of whom are different from those of the Western population. Given the high risk of CVD in Chinese postmenopausal population ${ }^{(9)}$, more evidence is needed to evaluate the association between ferritin and atherosclerosis in this population.

The use of high-resolution colour-coded duplex sonography offers the opportunity to assess the carotid intimamedia thickness (CIMT) and carotid plaque, as reliable markers of the severity of atherosclerosis ${ }^{(10)}$. Therefore, we attempted to investigate in the present study, the relationship between ferritin and carotid atherosclerosis in a communitybased population of Chinese postmenopausal women.

\section{Methods}

\section{Study population}

Postmenopausal women were recruited from the Changfeng Study, a community-based investigation of chronic diseases among middle-aged and elderly individuals, described elsewhere $^{(11)}$. From June 2009 to June 2012, a large group of 2717 postmenopausal women were initially enrolled for the study, from which 1539 participants were excluded for the following reasons: lack of physical examination and laboratory assessments ( $n$ 51); smoking or drinking ( $n$ 93); use of drugs known to influence Fe metabolism including the use of postmenopausal hormone therapy, mineral supplements (including Fe), vitamins and gluco-corticoid ( $n$ 280); prevalent CVD (myocardial infarction, stroke, or peripheral arterial disease) ( $n$ 172); prevalent haemodialysis ( $n 2)$; diagnosed liver disease, tumour, chronic inflammatory disease (such as rheumatoid arthritis and Crohn's disease), and/or a disorder of $\mathrm{Fe}$ metabolism (such as aplastic anaemia, b-thalassemia major and hemochromatosis) ( $n$ 203); diagnosed hypertension or the use of antihypertensive medications ( $n$ 634); diagnosed diabetes mellitus or the use of hypoglycaemic medications ( $n 70$ ); the use of lipid-lowering therapy or use of the anti platelet agents ( $n 33$ ); blood transfusion or donation in the past 3 months ( $n$ 1). Finally, after all these exclusions, a total of 1178 subjects were found eligible for the present investigation.

The study protocol, consent form, and subject-related materials were approved by the ethical committee of Zhongshan Hospital, Fudan University in accordance with the guidelines of the Declaration of Helsinki. We obtained informed consent from all women at the start of pre-baseline screening. Interviews, physical examinations and ultrasound scans were performed at the Changfeng Community Health Service Center.

\section{Clinical measurements}

Letters were sent to participants with instructions asking them not to alter their diet or level of physical activity for at least $3 \mathrm{~d}$ before the test. A questionnaire was administered by trained nurses to evaluate the medical history and lifestyle of each participant. Weight and height were measured while the participant was clothed in a light gown. The BMI was calculated as the weight divided by the height squared $\left(\mathrm{kg} / \mathrm{m}^{2}\right)$. The waist circumference was measured midway between the lowest rib margin and the iliac crest in a standing position, and the hip circumference was measured at the widest level over the greater trochanters. The waist:hip ratio (WHR) was calculated as the waist circumference divided by the hip circumference. The resting blood pressure was measured three times, and the mean value was used for the analysis. Blood samples were obtained after a fasting period of at least $10 \mathrm{~h}$. Total cholesterol, HDL-cholesterol, TAG and liver enzymes were measured using a model 7600 automated bio-analyser (Hitachi). The level of LDL-cholesterol was calculated using the Friedewald equation. The fasting blood glucose and 2-h glucose levels following a 75-g oral glucose challenge (PPG) for non-diabetics were measured using the glucose oxidase method. Serum ferritin and insulin were measured using an electrochemiluminescence immunoassay with an immunoassay analyser (Roche Cobas-6001; CV $<4.0$ and $<5.0 \%$, respectively). Early morning urine samples were analysed to determine the urinary albumin:creatinine ratio (UACR), employing an immunonephelometric method (CV $<4.0$ and $<3.2 \%$, respectively). $\mathrm{Hb}$ and leucocytes were measured from blood samples collected in EDTA-K2 $(3.6 \mathrm{mg})$ tubes (Becton, Dickinson and Company). These blood samples were analysed within $2 \mathrm{~h}$ of venepuncture, using an automatic blood counter (Sysmex XE-2100) (CV $<3.0$ and $<3.2 \%$, respectively). Homeostasis model assessment index for insulin resistance (HOMA-IR) and $\beta$-cell function (HOMA-B\%) were used to estimate insulin sensitivity and insulin secretion ${ }^{(12)}$.

The carotid arteries of the participants were evaluated by an experienced radiologist who was blinded to the participants' details using a GE Logic P5 (GE Healthcare) scanner with a $10 \mathrm{MHz}$ probe. The CIMT on both sides were measured in the common carotid artery approximately $1 \mathrm{~cm}$ proximal to the bifurcation at the far wall during end diastole. The CIMT was quantified at plaque-free sections of the carotid arteries, as the distance between the lumen-intima and mediaadventitia interfaces. Three values were measured on each side, and the average CIMT values were used for the analysis. The study procedure involved scanning the near and far walls of both the common carotid arteries, the carotid bifurcation, and the internal carotid artery. This scanning was done for detecting the presence of plaques, defined as the presence of focal wall thickening resulting in a thickness that is at least $50 \%$ greater than that of the surrounding vessel wall, 
or as a focal region with a CIMT greater than $1.5 \mathrm{~mm}$, protruding into the lumen, and distinct from the adjacent boundary, according to the American Society of Echocardiography ${ }^{(13)}$. Repeated measurements for the same subjects (performed in 104 subjects) yielded an intraclass correlation coefficient of 95\% (95\% CI 0.91, 0.97).

Hypertension was defined according to the Seventh Report of the Joint National Committee ${ }^{(14)}$. The diagnoses of impaired fasting glucose, impaired glucose tolerance, and diabetes mellitus were based on the American Diabetes Association 2010 criteria $^{(15)}$. The metabolic syndrome was defined according to the International Diabetes Federation 2005 guidelines $^{(16)}$. The diagnosis of CVD was based on self-reports and confirmed later through hospital medical records.

\section{Statistical analyses}

The data were expressed precisely in terms of the means, standard deviations and/or standard errors, frequencies or medians with 25 th and 75 th percentiles. Skewed variables were logarithmically transformed to improve normality prior to analysis. To evaluate the relationship between each parameter and the serum ferritin, the subjects were stratified according to the ferritin quartiles. The ranges of ferritin in the quartiles were $4 \cdot 1-102 \cdot 0,102 \cdot 2-158 \cdot 1,159 \cdot 2-226 \cdot 8$ and $226.9-591.0 \mathrm{ng} / \mathrm{ml}$. ANCOVA and logistic regression, with adjustments for CVD risk factors were conducted to compare means and proportions, respectively, across the ferritin quartiles. SPSS 16.0 for Windows (SPSS, Inc.) was used to perform the statistical analyses. All statistical tests were two tailed, and $P$ values less than 0.05 were considered significant.

\section{Results}

\section{Characteristics of the subjects according to the ferritin quartiles}

A total of 1178 postmenopausal women were evaluated. Table 1 outlines the anthropometric and biochemical parameters according to the quartiles groups for the ferritin analysis. Age, BMI, WHR, alanine transaminase, aspartate transaminase, $\gamma$-glutamyl transferase, total cholesterol, LDLcholesterol, HDL-cholesterol, TAG, fasting blood glucose, PPG, Hb, log HOMA-IR, log HOMA-B\% and the prevalence of the metabolic syndrome were found significantly associated

Table 1. Characteristics of the subjects according to quartile groups for serum ferritin

(Mean values and standard deviations; median values and interquartile ranges; number of subjects and percentages)

\begin{tabular}{|c|c|c|c|c|c|c|c|c|c|c|c|}
\hline \multirow[b]{2}{*}{ Variables } & \multicolumn{2}{|c|}{ All $(n 1178)$} & \multicolumn{2}{|c|}{$\begin{array}{l}\text { 1st quartile } \\
\text { (n 296) }\end{array}$} & \multicolumn{2}{|c|}{$\begin{array}{l}\text { 2nd quartile } \\
\qquad(n 293)\end{array}$} & \multicolumn{2}{|c|}{$\begin{array}{l}\text { 3rd quartile } \\
\text { (n 295) }\end{array}$} & \multicolumn{2}{|c|}{$\begin{array}{c}\text { 4th quartile } \\
\text { (n 294) }\end{array}$} & \multirow{2}{*}{$\begin{array}{c}P \text { (among } \\
\text { groups) }\end{array}$} \\
\hline & Mean & SD & Mean & SD & Mean & $\mathrm{SD}$ & Mean & SD & Mean & SD & \\
\hline Ferritin range (ng/ml) & \multicolumn{2}{|c|}{$4 \cdot 1-591 \cdot 0$} & \multicolumn{2}{|c|}{$4 \cdot 1-102 \cdot 0$} & \multicolumn{2}{|c|}{$102 \cdot 2-158 \cdot 1$} & \multicolumn{2}{|c|}{$159 \cdot 2-226 \cdot 8$} & \multicolumn{2}{|c|}{$226 \cdot 9-591 \cdot 0$} & \\
\hline Age (years) & $60 \cdot 8$ & 8.5 & $59 \cdot 1$ & $8 \cdot 7$ & $60 \cdot 2$ & $8 \cdot 2$ & $60 \cdot 8$ & $8 \cdot 1$ & $63 \cdot 2^{\star} \dagger \ddagger$ & 8.5 & $<0.001$ \\
\hline BMI $\left(\mathrm{kg} / \mathrm{m}^{2}\right)$ & $23 \cdot 5$ & $3 \cdot 2$ & $23 \cdot 0$ & $3 \cdot 3$ & $23 \cdot 0$ & $3 \cdot 1$ & $23 \cdot 6^{*} \dagger$ & 2.9 & $24 \cdot 3^{\star} \dagger \ddagger$ & 3.2 & $<0.001$ \\
\hline WHR & 0.867 & 0.069 & 0.854 & 0.063 & 0.863 & 0.070 & $0.867^{*}$ & 0.061 & $0.885^{\star} \dagger \ddagger$ & 0.076 & $<0.001$ \\
\hline $\mathrm{SBP}(\mathrm{mmHg})$ & $127 \cdot 9$ & $16 \cdot 8$ & $127 \cdot 1$ & $16 \cdot 4$ & $127 \cdot 8$ & $17 \cdot 0$ & $126 \cdot 6$ & $15 \cdot 5$ & $129 \cdot 9^{\star} \ddagger$ & $18 \cdot 0$ & 0.08 \\
\hline $\mathrm{DBP}(\mathrm{mmHg})$ & $73 \cdot 0$ & 8.7 & 73.0 & 8.5 & $73 \cdot 0$ & 8.9 & $73 \cdot 0$ & $8 \cdot 6$ & $73 \cdot 1$ & 8.7 & 0.995 \\
\hline $\operatorname{ALT}(\mathrm{U} / \mathrm{I})$ & $18 \cdot 7$ & $9 \cdot 1$ & $16 \cdot 1$ & 8.9 & $17 \cdot 0$ & $7 \cdot 5$ & $18 \cdot 1$ & $7 \cdot 6$ & $23 \cdot 7^{\star} \dagger \ddagger$ & $8 \cdot 5$ & $<0.001$ \\
\hline AST (U/I) & $22 \cdot 1$ & $8 \cdot 1$ & $20 \cdot 7$ & 6.5 & $21 \cdot 3$ & $5 \cdot 2$ & $21 \cdot 2$ & 6.5 & $25 \cdot 1^{*} \dagger \ddagger$ & $8 \cdot 0$ & $<0.001$ \\
\hline$\gamma-\mathrm{GT}(\mathrm{U} / \mathrm{I})$ & $27 \cdot 3$ & $17 \cdot 1$ & $23 \cdot 2$ & $18 \cdot 0$ & 23.9 & $16 \cdot 5$ & 25.9 & $11 \cdot 1$ & 36.5 & $27 \cdot 4$ & 0.002 \\
\hline $\mathrm{TC}(\mathrm{mmol} / \mathrm{l})$ & $5 \cdot 3$ & 0.9 & $5 \cdot 1$ & 0.8 & $5 \cdot 4^{*}$ & 0.8 & $5 \cdot 3^{*}$ & 0.9 & $5 \cdot 5^{\star}$ & $1 \cdot 0$ & $<0.001$ \\
\hline LDL-C (mmol/l) & $3 \cdot 1$ & 0.8 & 2.9 & 0.7 & $3 \cdot 1^{*}$ & 0.7 & $3 \cdot 1^{*}$ & 0.7 & $3 \cdot 2^{\star}$ & 0.9 & $<0.001$ \\
\hline HDL-C (mmol/l) & 1.6 & 0.4 & 1.6 & 0.4 & 1.6 & 0.4 & $1 \cdot 5^{\star}$ & 0.4 & $1 \cdot 5^{\star} \dagger \ddagger$ & 0.4 & $<0.001$ \\
\hline $\mathrm{TAG}(\mathrm{mmol} / \mathrm{l})$ & 1.5 & 0.7 & $1 \cdot 3$ & 0.6 & $1.4^{*}$ & 0.6 & $1 \cdot 6^{*}$ & 0.8 & $1 \cdot 5^{\star} \dagger \ddagger$ & 0.4 & $<0.001$ \\
\hline $\mathrm{FBG}(\mathrm{mmol} / \mathrm{l})$ & $5 \cdot 2$ & 0.8 & $5 \cdot 1$ & 0.9 & $5 \cdot 2$ & 0.7 & $5 \cdot 2$ & 0.8 & $5 \cdot 4^{*} \dagger$ & 0.9 & 0.006 \\
\hline $\mathrm{PPG}(\mathrm{mmol} / \mathrm{l})$ & $7 \cdot 7$ & $3 \cdot 2$ & $6 \cdot 4$ & $2 \cdot 3$ & 6.5 & 2.5 & $7 \cdot 0^{*} \dagger$ & 2.5 & $7 \cdot 7^{*} \dagger \ddagger$ & $3 \cdot 2$ & $<0.001$ \\
\hline $\mathrm{Hb}(\mathrm{g} / \mathrm{l})$ & $133 \cdot 7$ & $9 \cdot 5$ & $131 \cdot 6$ & 11.4 & $133 \cdot 3$ & $8 \cdot 8$ & 134.9 & $8 \cdot 0$ & $135 \cdot 0$ & $9 \cdot 2$ & $<0.001$ \\
\hline Leucocytes $\left(10^{9} / \mathrm{l}\right)$ & $5 \cdot 3$ & $1 \cdot 3$ & $5 \cdot 3$ & 1.4 & $5 \cdot 3$ & $1 \cdot 2$ & $5 \cdot 3$ & $1 \cdot 2$ & 5.5 & 1.4 & 0.067 \\
\hline UACR range $(\mu \mathrm{g} / \mathrm{mg})$ & & & & & & & & & & & 0.648 \\
\hline Median & \multicolumn{2}{|c|}{$6 \cdot 4$} & \multicolumn{2}{|c|}{$6 \cdot 1$} & \multicolumn{2}{|c|}{$6 \cdot 3$} & \multicolumn{2}{|c|}{6.5} & \multicolumn{2}{|c|}{$6 \cdot 7$} & \\
\hline Interquartile range & \multicolumn{2}{|c|}{$4 \cdot 3-9 \cdot 9$} & \multicolumn{2}{|c|}{$4 \cdot 3-9 \cdot 5$} & \multicolumn{2}{|c|}{$4 \cdot 3-9 \cdot 5$} & \multicolumn{2}{|c|}{$4.2-9.9$} & \multicolumn{2}{|c|}{$4 \cdot 3-10 \cdot 5$} & \\
\hline HOMA-IR & \multirow{2}{*}{\multicolumn{4}{|c|}{1.7}} & \multirow{2}{*}{\multicolumn{2}{|c|}{$1.6^{*}$}} & & & & & $<0.001$ \\
\hline Median & & & \multirow{2}{*}{\multicolumn{2}{|c|}{$\begin{array}{c}1 \cdot 4 \\
0.9-2 \cdot 1\end{array}$}} & & & \multicolumn{2}{|c|}{$1 \cdot 8^{*} \dagger$} & \multicolumn{2}{|c|}{$2 \cdot 0^{\star} \dagger \ddagger$} & \\
\hline Interquartile range & \multicolumn{2}{|c|}{$\begin{array}{c}1 \cdot 1 \\
1 \cdot 2-2 \cdot 5\end{array}$} & & & $1 \cdot 1$ & & $1 \cdot 2$ & & $1 \cdot 5-3$ & & \\
\hline HOMA-B\% & & & & & & & & & & & $<0.001$ \\
\hline Median & & & & & & & 95 & & $110 \cdot 7$ & & \\
\hline Interquartile range & $69 \cdot 3$ & $30 \cdot 3$ & $64 \cdot 1-$ & $17 \cdot 0$ & 64.4 & $21 \cdot 6$ & $68 \cdot 9-$ & $35 \cdot 0$ & $78 \cdot 7-1$ & 1.7 & \\
\hline Metabolic syndrome & & & & & & & & & & & $<0.001$ \\
\hline$n$ & & & & & & & 64 & & $106^{*}$ & & \\
\hline$\%$ & & & & & & & 24 & & 39 & & \\
\hline
\end{tabular}

WHR, waist:hip ratio; SBP, systolic blood pressure; DBP, diastolic blood pressure; ALT, alanine transaminase; AST, aspartate transaminase; $\gamma$-GT, $\gamma$-glutamyl transferase; TC, total cholesterol; LDL-C, LDL-cholesterol; HDL-C, HDL-cholesterol; FBG, fasting blood glucose; PPG, 2-h blood glucose following a 75-g oral glucose challenge; UACR, urine albumin:creatinine ratio; HOMA-IR, homeostasis model assessment index for insulin resistance; HOMA-B, homeostasis model assessment index for $\beta$-cell function.

${ }^{*}$ ANOVA with least significant difference post hoc test or $\chi^{2}$ statistical analysis: $P<0.05 \mathrm{v}$. 1st quartile.

† ANOVA with least significant difference post hoc test or $\chi^{2}$ statistical analysis: $P<0.05 v$. 2nd quartile.

$\ddagger$ ANOVA with least significant difference post hoc test or $\chi^{2}$ statistical analysis: $P<0.05 v$. 3rd quartile. 
Table 2. Association of the anthropometric and biochemical parameters with ferritin in postmenopausal women ( $\beta$ Coefficients and $95 \%$ confidence intervals)

\begin{tabular}{|c|c|c|c|c|}
\hline & $\beta$ & $95 \% \mathrm{Cl}$ & Standardised $\beta$ & $P$ \\
\hline Age (per 1 year) & 3.003 & $2.134,3.931$ & 0.190 & $<0.001$ \\
\hline BMI (per 1 unit) & 7.525 & $5 \cdot 106,9.943$ & 0.175 & $<0.001$ \\
\hline WHR & 301.992 & $190 \cdot 204,413 \cdot 779$ & 0.153 & $<0.001$ \\
\hline SBP (per $1 \mathrm{mmHg}$ ) & 0.292 & $0.171,0.775$ & 0.036 & 0.216 \\
\hline DBP (per $1 \mathrm{mmHg}$ ) & -0.369 & $1.265,0.527$ & -0.024 & 0.419 \\
\hline $\operatorname{ALT}(1 \mathrm{U} / \mathrm{l})$ & 1.951 & $1.483,2.418$ & 0.232 & $<0.001$ \\
\hline AST (1 U/I) & $2 \cdot 749$ & $2.029,3.470$ & 0.213 & $<0.001$ \\
\hline$\gamma$-GT $(1 \mathrm{U} / \mathrm{l})$ & 0.416 & $0.252,0.579$ & 0.144 & $<0.001$ \\
\hline TC $(1 \mathrm{mmol} / \mathrm{l})$ & 14.575 & $5 \cdot 830,23 \cdot 320$ & 0.095 & 0.001 \\
\hline LDL-C (1 mmol//) & $12 \cdot 636$ & $2 \cdot 478,22 \cdot 794$ & 0.071 & 0.015 \\
\hline HDL-C (1 mmol/li) & -41.547 & $62 \cdot 302,20 \cdot 793$ & -0.114 & $<0.001$ \\
\hline TAG $(1 \mathrm{mmol} / \mathrm{l})$ & $22 \cdot 627$ & $15 \cdot 279,29 \cdot 976$ & 0.174 & $<0.001$ \\
\hline FBG $(1 \mathrm{mmol} / \mathrm{l})$ & $20 \cdot 797$ & $11.626,29.967$ & 0.129 & $<0.001$ \\
\hline PPG (1 mmol/l) & $10 \cdot 650$ & $7.803,13.496$ & 0.210 & $<0.001$ \\
\hline $\mathrm{Hb}(1 \mathrm{~g} / \mathrm{l})$ & 1.637 & $0.826,2.448$ & 0.115 & $<0.001$ \\
\hline Leucocytes $\left(1 \times 10^{9} / l\right)$ & 3.337 & $2.608,9 \cdot 281$ & 0.032 & 0.271 \\
\hline $\log$ UACR & -1.055 & $20.726,18.617$ & -0.003 & 0.916 \\
\hline log HOMA-IR & $142 \cdot 357$ & $113.818,170.895$ & 0.275 & $<0.001$ \\
\hline log HOMA-B\% & $95 \cdot 727$ & $62.959,128.495$ & 0.165 & $<0.001$ \\
\hline Metabolic syndrome & $55 \cdot 127$ & $36 \cdot 817,73.438$ & 0.170 & $<0.001$ \\
\hline
\end{tabular}

WHR, waist:hip ratio; SBP, systolic blood pressure; DBP, diastolic blood pressure; ALT, alanine transaminase; AST, aspartate transaminase; $\gamma$-GT, $\gamma$-glutamyl transferase; TC, total cholesterol; LDL-C, LDL-cholesterol; HDL-C, HDL-cholesterol; FBG, fasting blood glucose; PPG, 2-h blood glucose following a 75-g oral glucose challenge; UACR, urine albumin-to-creatinine; HOMA-IR, homeostasis model assessment index for insulin resistance; HOMA-B, homeostasis model assessment index for $\beta$-cell function.

with the ferritin quartiles, when the traditional CVD risk factors were examined.

\section{Association of the anthropometric and biochemical} parameters with serum ferritin levels

Correlations between serum ferritin and anthropometric indicators for women are displayed in Table 2. Linear regression analysis showed an association between serum ferritin and age, BMI, WHR, alanine transaminase, aspartate transaminase, $\boldsymbol{\gamma}$-glutamyl transferase, total cholesterol, LDL-cholesterol, HDL-cholesterol, TAG, fasting blood glucose, PPG, Hb, log HOMA-IR, log HOMA-B\% and the metabolic syndrome. Multivariate linear stepwise regression analysis was performed to evaluate the independent factors of serum ferritin. The analysis demonstrated that age (standardised $\beta=0 \cdot 233, P<0 \cdot 001$ ), alanine transaminase (standardised $\beta=0 \cdot 194, P<0 \cdot 001$ ), log HOMA-IR (standardised $\quad \beta=0.181, \quad P<0.001), \quad$ TAG $\quad$ (standardised $\beta=0.083, P=0.003$ ), Hb (standardised $\beta=0.080, P=0.004$ ), PPG (standardised $\beta=0.079, P=0.009$ ) and systolic blood pressure (standardised $\beta=-0 \cdot 135, P<0.001$ ) were independently associated with serum ferritin.

\section{Association between carotid atherosclerosis and serum ferritin levels}

Table 3 presents the CIMT of the subjects according to ferritin quartile groups. Compared with the subjects in the first ferritin quartile, those in the third and fourth quartiles had significantly increased CIMT. After adjusting for conventional CVD risk factors, $\mathrm{Hb}$, leucocytes, log UACR and liver enzymes, the subjects with elevated ferritin levels in the fourth quartile had still greater CIMT than those in the first quartile.

Table 3. Carotid intima-media thickness (CIMT) in the subjects according to quartile groups for ferritin

(Mean values with their standard errors)

\begin{tabular}{|c|c|c|c|c|c|c|c|c|c|}
\hline & \multicolumn{9}{|c|}{ Quartile groups for ferritin } \\
\hline & \multicolumn{2}{|c|}{ 1st quartile } & \multicolumn{2}{|c|}{ 2nd quartile } & \multicolumn{2}{|c|}{ 3rd quartile } & \multicolumn{2}{|c|}{ 4th quartile } & \multirow[b]{2}{*}{$P$} \\
\hline & Mean & SE & Mean & SE & Mean & SE & Mean & SE & \\
\hline \multicolumn{10}{|l|}{ Unadjusted } \\
\hline $\mathrm{CIMT}(\mathrm{mm})$ & 0.707 & 0.008 & 0.712 & 0.008 & $0.729^{*}$ & 0.008 & $0.733^{\star} \dagger$ & 0.008 & 0.011 \\
\hline \multicolumn{10}{|l|}{ Model 回 } \\
\hline CIMT (mm) & 0.713 & 0.007 & 0.716 & 0.007 & 0.718 & 0.007 & $0.731^{*}$ & 0.007 & 0.037 \\
\hline
\end{tabular}

* ANOVA with least significant difference post hoc test: $P<0.05 v$. 1st quartile.

† ANOVA with least significant difference post hoc test: $P<0.05 v$. 2nd quartile.

¥Model [?: adjusting for age, fasting blood glucose, PPG (2-h blood glucose following a 75-g oral glucose challenge), leucocytes, $\gamma$-glutamyl transferase, log (urine albumin-to-creatinine), BMI, waist:hip ratio, systolic blood pressure, diastolic blood pressure, alanine transaminase, aspartate transaminase, HDL-cholesterol, LDL-cholesterol, log homeostasis model assessment index for insulin resistance, log homeostasis model assessment index for $\beta$-cell function and the metabolic syndrome. 


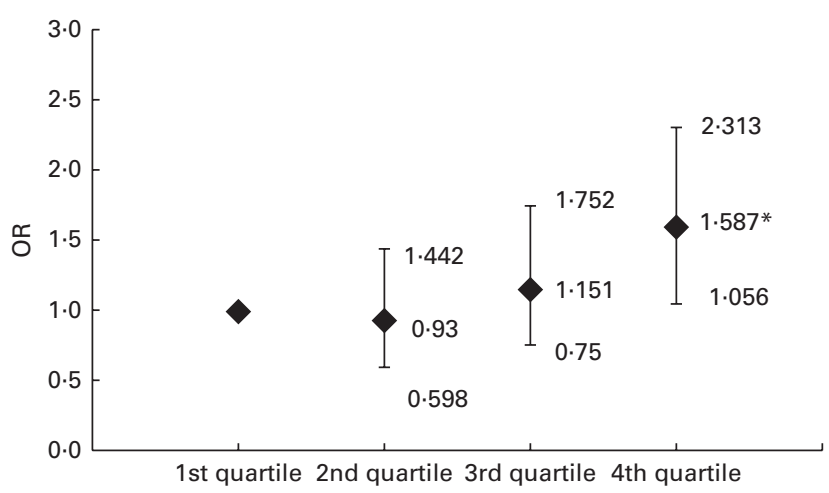

Fig. 1. Risk of carotid plaque in the subjects across the ferritin quartiles. Values are OR, with $95 \% \mathrm{Cl}$ represented by vertical bars. OR of carotid plaque in the subjects according to ferritin quartile groups are after adjusting for age, BMI, waist:hip ratio, fasting blood glucose, 2-h glucose levels following a $75-\mathrm{g}$ oral glucose challenge, $\mathrm{Hb}$, leucocytes, log urine albumin: creatinine ratio, systolic blood pressure, diastolic blood pressure, TAG, HDL-cholesterol, LDL-cholesterol, alanine transaminase, aspartate transaminase, $\gamma$-glutamyl transferase, log homeostasis model assessment index for insulin resistance, log homeostasis model assessment index for $\beta$-cell function and the prevalence of the metabolic syndrome, respectively. *ANOVA with logistic regression analysis: $P<0.05$ compared with first, second and third quartiles.

Compared with the subjects in the first, second and third ferritin quartiles, those in the fourth quartile had significantly higher prevalence of carotid plaque $(22 \cdot 2,20.9$ and $24.5 \mathrm{v}$. $32.0 \%$, respectively, $P=0.023$ ). After adjusting for conventional CVD risk factors, $\mathrm{Hb}$, leucocytes, log UACR and liver enzymes, the subjects with elevated levels of ferritin in the fourth quartile had 1.587 -fold increased risk for carotid plaques relative to those in the lowest quartile (Fig. 1).

\section{Discussion}

The present study showed that serum ferritin was independently associated with carotid atherosclerosis in Chinese postmenopausal women. The CIMT and the prevalence of carotid plaques significantly increased in the subjects with increasing ferritin, after adjusting for conventional CVD risk factors, $\mathrm{Hb}$, leucocytes, log UACR and liver enzymes.

Although the iron-heart disease hypothesis has been given attention in recent years, the epidemiological findings are incongruent. Serum ferritin is considered the best single indicator of total body $\mathrm{Fe}$ stores $^{(17)}$. The relationship of serum ferritin with early CVD, particularly atherosclerosis, has not been evaluated extensively. A significant positive association between serum ferritin and subclinical atherosclerosis was reported in some studies ${ }^{(6,17)}$, whereas a few others did not find any association ${ }^{(7,8,18)}$. In evaluating these studies, a few considerations must be taken into account. As mentioned in previous studies ${ }^{(17,19-21)}$, the use of ferritin in assessing body Fe stores has been somewhat challenging, because ferritin levels can be elevated due to inflammation, cancer and liver disease as well ${ }^{(22)}$. Serum ferritin concentration can also be increased in some conditions like obesity and smoking which are associated risk factors for $\mathrm{CVD}^{(23)}$. It becomes thus difficult to determine whether the association of ferritin with
CVD is due to increased serum ferritin levels or other concomitant conditions. In addition, serum ferritin has been correlated with dyslipidemia biomarkers, diabetes and hepatic enzymes ${ }^{(24)}$. Thus, adjustments for these components become very important. Moreover, multiple studies have shown that excess body $\mathrm{Fe}$ is associated with one or more components of the metabolic syndrome ${ }^{(25,26)}$. We controlled possible confounding variables in the study by several ways. First, we restricted the analysis to subjects without CVD-associated risk factors including known hypertension and diabetes. In addition, we excluded subjects: with inflammation involving smoking, CVD, liver disease, tumour and chronic inflammatory disease; undergoing treatment for diabetes, hypertension and dyslipidaemia; using antiplatelet agents. Finally, in our statistical analyses, we adjusted for as many factors of influence as possible, including BMI, WHR, blood pressure, $\mathrm{Hb}$, leucocytes, UACR, fasting and 2-h blood glucose from the oral glucose tolerance test, lipid profiles, liver enzymes, insulin resistance and the prevalence of the metabolic syndrome. When these factors were added to the multivariate-adjusted models, the results of the association of carotid atherosclerosis with serum ferritin, however, were found unchanged. The residual confounding due to other factors such as dietary regimen and physical activity must have also been considered. Because the present study focused on subclinical atherosclerosis among apparently healthy women, we presumed that dietary regimen and physical activity would not affect the results. Thus, the association between ferritin and carotid atherosclerosis was a more compelling assumption.

The possible effects of body Fe stores on CHD risk may be viewed as direct or indirect. In the present study, ferritin was positively associated with age, BMI, HOMA-IR, TAG and PPG. These findings were also supported by other studies ${ }^{(23-26)}$. Therefore, the effects of ferritin on atherosclerosis might be due to its ability to affect the glucose and lipid metabolism, and other metabolic factors. The exact molecular mechanism of Fe-related pathology in the metabolic syndrome and diabetes is not clearly understood. Fe is a powerful pro-oxidant and can cause pancreatic $\beta$-cell damage by producing reactive oxygen species. Thus, Fe deposition in $\beta$-cells can lead to apoptosis and consequently to decreased insulin synthesis and secretion ${ }^{(27,28)}$. A recent study of 492 subjects observed associations of several markers of Fe metabolism with adipocyte IR and adiponectin; implication was that the factors related to $\mathrm{Fe}$ may contribute to adipocyte IR early in the pathogenesis of type 2 diabetes mellitus ${ }^{(29)}$. On the other hand, we demonstrated in the present study a positive and independent association between ferritin and carotid atherosclerosis, after adjustment for established CVD risk factors, $\mathrm{Hb}$, leucocytes, log UACR and liver function in postmenopausal Chinese women, conforming to strict inclusion criteria. If ferritin and the other risk factors share a common causal pathway, adjusting for these risk factors may attenuate the relationship between ferritin and carotid atherosclerosis. However, ferritin remained a relatively strong predictor after full adjustment in the present study, suggesting that there was an independent additive component in the relationship between ferritin and CVD. Therefore, other atherogenic mechanisms 
could conceivably be involved. First, ferritin can act as a catalyser in the production of oxygen free radicals, and the promotion of lipid peroxidation by reducing the levels of antioxidants in plasma. Ferritin can play a role in the formation of oxidised $\mathrm{LDL}^{(30,31)}$. When the LDL-cholesterol molecules become oxidised, they can be highly cardiotoxic; this is because they may cause direct endothelial injury or enhance the uptake of LDL by the macrophages, leading to the generation of foam cells $^{(2)}$. Ferritin had been described in human atherosclerotic lesions and in diseased coronary arteries from patients with coronary artery disease ${ }^{(17)}$. Localisation of ferritin in the arteries may contribute to the oxidation of lipids within the vessel wall, and formation of oxidised LDL, leading to the development of foam cells, and progression of atherosclerosis ${ }^{(32)}$. Third, it has been suggested that body Fe stores might directly lead to CHD through a mechanism which might involve oxidative damage to the myocardium ${ }^{(33)}$. Mechanisms other than atherosclerosis could be responsible for the harmful effect of Fe, such as mechanisms that involve the thrombotic pathway ${ }^{(34)}$, or decreased insulin sensitivity ${ }^{(35)}$.

The present results suggest that ferritin is a marker of or risk factor for atherosclerosis. Ferritin could be adopted as an additional marker for the detection of CVD. In the context of patient care, screening that is based on ferritin would enable clinicians to provide early interventions. An early evaluation of ferritin would be advantageous for the early detection of CVD, and individuals with increased ferritin might benefit from more aggressive follow-up lifestyle modifications and dietary regimen.

As examined above, serum ferritin levels are found associated with CVD. Then can appropriate interventions beneficially impact Fe-reduced CVD risk? Unfortunately, only a limited number of intervention studies on this question are available in literature, and the outcomes remain controversial. A multicentre, randomised, controlled, single-blinded clinical trial based on the $\mathrm{Fe}$ and Atherosclerosis Study (FeAST) showed that the reduction of body Fe stores in patients with symptomatic peripheral arterial disease did not significantly decrease all-cause mortality or death plus nonfatal myocardial infarction and stroke ${ }^{(36)}$. Another study suggests that modulation of $\mathrm{Fe}$ can reduce cardiovascular risk ${ }^{(37)}$. In the recent randomised controlled single blinded study, Zacharski et $a{ }^{(37)}$ demonstrated that lower $\mathrm{Fe}$ burden predicted improved outcomes overall and was enhanced by phlebotomy. Controlling Fe burden may improve survival and prevent or delay nonfatal myocardial infarction and stroke in young patients with peripheral artery disease.

Apart from serum ferritin, other laboratory parameters such as catalytic $\mathrm{Fe}^{(38)}$, transferrin saturation ${ }^{(8,17)}$, and soluble transferrin receptor $(\mathrm{sTfR})^{(8,17)}$ have been used to assess the body Fe stores and their potential association with coronary artery disease. Among them, ferritin and sTfR are considered to be the most reliable estimates of Fe status; ferritin is considered the best measure of body Fe stores, whereas STfR is considered to reflect the functional Fe compartment. Serum ferritin is the most widely used biomarker of body Fe stores in epidemiological studies, despite being shown to be affected by inflammation status. Conversely, sTfR is not altered by inflammatory processes ${ }^{(39)}$, and its levels in blood are proportional to the cell requirements for $\mathrm{Fe}^{(40)}$. Increased $\mathrm{Fe}$ amount due to higher density of transferrin receptor in cell membranes may facilitate tissue damage through generation of free radicals and lipid peroxidation and potentiate the progression of atherosclerotic lesions in arterial walls. The increased synthesis of transferrin receptor may be a consequence of action of various mediators acting in the atherosclerotic lesions. In addition, Fe status per se may not dictate susceptibility to injury but rather to Fe that is catalytically available to participate in free radical reactions ${ }^{(38)}$.

Our findings need to be considered in the context of certain limitations. It is admittedly cross-sectional, and there may be measurement errors due to within-person variability in ferritin, and reliance on a single measure of the biomarkers in the current analysis. The cross-sectional nature of this study limits our ability to determine causality. However, the temporal relationship between ferritin and carotid atherosclerosis has been well established. Our analyses were adjusted for important confounders, after excluding subjects with any other disease that may be the cause of elevated ferritin. Our participants were postmenopausal women, and therefore, the results cannot be applied to younger subjects. In the present study only a single marker for Fe homeostasis had been analysed. However, Fe metabolism is complex, and a single marker may provide insufficient information. It is also known that several markers of inflammation such as C-reactive protein as well as malnutrition are associated with CHD. However, we do not have data on C-reactive protein and serum albumin. Hence, the association between Fe metabolism and carotid atherosclerosis in Chinese women should be confirmed using a larger sample and prospective studies.

\section{Conclusion}

In conclusion, we demonstrated that ferritin has a relatively strong positive correlation with carotid atherosclerosis even after adjusting for conventional CVD risk factors, $\mathrm{Hb}$, leucocytes, $\log$ UACR and liver function in postmenopausal Chinese women. Our findings suggest that individuals with increased ferritin levels require aggressive management of CVD risk factors. Causality should be affirmed by ongoing as well as future studies., Also effective dietary regimen for decreased serum ferritin status should be recommended to postmenopausal Chinese women which would ultimately lower the risk of CVD.

\section{Acknowledgements}

The Shanghai Changfeng Study has received great support from Changfeng Health Center, the Health Bureau of Putuo District, and the committees of all the sub-communities of Changfeng. The contributions of all the working staffs and participants of the study are greatly appreciated and acknowledged.

This work was supported by grants made available to X. G. from the Major State Basic Research Development Program of China (2012CB524906 http://www.973.gov.cn/Default_3. aspx), National Natural Science Foundation of China 
(81270933), and Shanghai Municipal Health Bureau Foundation (12GWZX0103 and 2013ZYJB0802).

The authors' contributions are as follows: H. M., H. L., Y. H., X. L., W. H., X. J., J. G., N. Z., B. S., B. P. and X. G. conceived and designed the experiments; H. M., H. L., X. L., W. H. and J. G. performed the experiments; H. M., N. Z. and J. G. analysed the data; H. M. and X. G. wrote the paper. All authors read and approved the final manuscript.

The authors declare that they have no conflict of interests.

\section{References}

1. Boukhris M, Tomasello SD, Marzà F, et al. (2014) Coronary heart disease in postmenopausal women with type II diabetes mellitus and the impact of estrogen replacement therapy: a narrative review. Int J Endocrinol 2014, 413920.

2. Masse PG, Dosy J, Cole DEC, et al. (2004) Is serum ferritin an additional cardiovascular risk factor for all postmenopausal women? Ann Nutr Metab 48, 381-389.

3. Sullivan JL (1981) Iron and the sex difference in heart disease risk. Lancet i, 1293-1294.

4. Salonen JT, Nyyssonen K, Korpela H, et al. (1992) High stored iron levels are associated with excess risk of myocardial infarction in eastern Finnish men. Circulation 86 803-811.

5. van der A DL, Grobbee DE, Roest M, et al. (2005) Serum ferritin is a risk factor for stroke in postmenopausal women. Stroke 36, 1637-1641.

6. Wolff B, Völzke H, Lüdemann J, et al. (2004) Association between high serum ferritin levels and carotid atherosclerosis in the study of health in Pomerania (SHIP). Stroke 35, 453-457.

7. Galan P, Noisette N, Estaquio C, et al. (2006) Serum ferritin, cardiovascular risk factors and ischaemic heart diseases: a prospective analysis in the SU.VI.MAX (SUpplementation en VItamines et Minéraux AntioXydants) cohort. Public Health Nutr 9, 70-74.

8. Menke A, Muntner P, Fernández-Real JM, et al. (2012) The association of biomarkers of iron status with mortality in US adults. Nutr Metab Cardiovasc Dis 22, 734-740.

9. Mosca L, Barrett-Connor E \& Wenger NK (2011) Sex/gender differences in cardiovascular disease prevention: what a difference a decade makes. Circulation 124, 2145-2154.

10. Ma H, Lin H, Hofman A, et al. (2013) Low-grade albuminuria is associated with carotid atherosclerosis in normotensive and euglycemic Chinese middle-aged and elderly adults: the Shanghai Changfeng Study. Atherosclerosis 228, $237-242$

11. Gao X, Hofman A, Hu Y, et al. (2010) The Shanghai Changfeng Study: a community based prospective cohort study of chronic diseases among middle-aged and elderly: objectives and design. Eur J Epidemiol 25, 885-893.

12. Matthews DR, Hosker JP, Rudenski AS, et al. (1985) Homeostasis model assessment: insulin resistance and beta-cell function from fasting plasma glucose and insulin concentrations in man. Diabetologia 28, 412-419.

13. Stein JH, Korcarz CE, Hurst RT, et al. (2008) Use of carotid ultrasound to identify subclinical vascular disease and evaluate cardiovascular disease risk: a consensus statement from the American Society of Echocardiography Carotid IntimaMedia Thickness Task Force. Endorsed by the Society for Vascular Medicine. J Am Soc Echocardiogr 21, 93-111.

14. Chobanian AV, Bakris GL, Black HR, et al. (2003) Seventh report of the joint national committee on prevention, detection, evaluation, and treatment of high blood pressure. Hypertension 42, 1206-1252.

15. American Diabetes Association (2010) Standards of medical care in diabetes-2010. Diabetes Care 33, S11-S61.

16. Pemminati S, Prabha Adhikari MR, Pathak R, et al. (2010) Prevalence of metabolic syndrome (METS) using IDF 2005 guidelines in a semi urban south Indian (Boloor Diabetes Study) population of Mangalore. J Assoc Physicians India 58, 674-677.

17. Ahluwalia N, Genoux A, Ferrieres J, et al. (2010) Iron status is associated with carotid atherosclerotic plaques in middleaged adults. J Nutr 140, 812-816.

18. van der A DL, Marx JJ, Grobbee DE, et al. (2006) Nontransferrin-bound iron and risk of coronary heart disease in postmenopausal women. Circulation 113, 1942-1949.

19. Eftekhari MH, Mozaffari-Khosravi H, Shidfar F, et al. (2013) Relation between body iron status and cardiovascular risk factors in patients with cardiovascular disease. Int J Prev Med 4, 911-916.

20. Crist BL, Alekel DL, Ritland LM, et al. (2009) Association of oxidative stress, iron, and centralized fat mass in healthy postmenopausal women. $J$ Womens Health (Larchmt) 18, 795-801.

21. Alissa EM, Ahmed WH, Al-Ama N, et al. (2007) Relationship between indices of iron status and coronary risk factors including diabetes and the metabolic syndrome in Saudi subjects without overt coronary disease. J Trace Elem Med Biol 21, 242-254.

22. Wang W, Knovich MA, Coffman LG, et al. (2010) Serum ferritin: past, present and future. Biochim Biophys Acta 1800, 760-769.

23. Lee CC, Adler AI, Sandhu MS, et al. (2009) Association of C-reactive protein with type 2 diabetes: prospective analysis and meta-analysis. Diabetologia 52, 1040-1047.

24. Basuli D, Stevens RG, Torti FM, et al. (2014) Epidemiological associations between iron and cardiovascular disease and diabetes. Front Pharmacol 20, 117.

25. González AS, Guerrero DB, Soto MB, et al. (2006) Metabolic syndrome, insulin resistance and the inflammation markers C-reactive protein and ferritin. Eur J Clin Nutr 60 , 802-809.

26. Sun L, Franco OH, Hu FB, et al. (2008) Ferritin concentrations, metabolic syndrome, and type 2 diabetes in middle-aged and elderly Chinese. J Clin Endocrinol Metab 93, 4690-4696.

27. Ferrannini E (2000) Insulin resistance, iron, and the liver. Lancet 355, 2181-2182.

28. Cooksey RC, Jouihan HA, Ajioka RS, et al. (2004) Oxidative stress, beta-cell apoptosis, and decreased insulin secretory capacity in mouse models of hemochromatosis. Endocrinology 145, 5305-5312.

29. Wlazlo N, van Greevenbroek MM, Ferreira I, et al. (2013) Iron metabolism is associated with adipocyte insulin resistance and plasma adiponectin: the Cohort on Diabetes and Atherosclerosis Maastricht (CODAM) study. Diabetes Care 36, 309-315.

30. Balla J, Vercellotti GM, Jeney V, et al. (2007) Heme, heme oxygenase, and ferritin: how the vascular endothelium survives (and dies) in an iron-rich environment. Antioxid Redox Signal 9, 2119-2137.

31. Liao X, Lv C, Zhang X, et al. (2012) A novel strategy of natural plant ferritin to protect DNA from oxidative damage during iron oxidation. Free Radic Biol Med 53, $375-382$. 
32. Yuan XM, Brunk UT \& Olsson AG (1995) Effects of iron- and hemoglobin loaded human monocyte-derived macrophages on oxidation and uptake of LDL. Arterioscler Thromb Vasc Biol 15, 1345-1351.

33. Sempos CT \& Looker AC (2001) Iron status and the risk of coronary heart disease: an example of the use of nutritional epidemiology in chronic disease research. J Nutr Biochem 12, 170-182

34. Day SM, Duquaine D, Mundada LV, et al. (2003) Chronic iron administration increases vascular oxidative stress and accelerates arterial thrombosis. Circulation 107, 2601-2606.

35. Engberink MF, Geleijnse JM, Durga J, et al. (2008) Blood donation, body iron status and carotid intima-media thickness. Atherosclerosis 196, 856-862.
36. Zacharski LR, Chow BK, Howes PS, et al. (2007) Reduction of iron stores and cardiovascular outcomes in patients with peripheral arterial disease: a randomized controlled trial. JAMA 297, 603-610.

37. Zacharski LR, Shamayeva G \& Chow BK (2011) Effect of controlled reduction of body iron stores on clinical outcomes in peripheral arterial disease. Am Heart J 162, 949-957.e1.

38. Rajapurkar MM, Shah SV, Lele SS, et al. (2012) Association of catalytic iron with cardiovascular disease. Am J Cardiol 109, 438-442.

39. Désidéri-Vaillant C, Galinat H, Sapin-Lory J, et al. (2011) Serum transferrin receptor in the assessment of iron status. Transfus Clin Biol 18, 36-39.

40. Speeckaert MM, Speeckaert R \& Delanghe JR (2010) Biological and clinical aspects of soluble transferrin receptor. Crit Rev Clin Lab Sci 47, 213-228. 